\title{
LA-UR-21-27912
}

Approved for public release; distribution is unlimited.

Title: $\quad$ INECP Country Engagement Plans and Transformational Events

Author(s): $\quad$ Gonzalez, Shane Michael

Intended for: $\quad$ NEN Summer Student Symposium, Keepin School Student Talks

Issued: $\quad$ 2021-08-06 
Disclaimer:

Los Alamos National Laboratory, an affirmative action/equal opportunity employer, is operated by Triad National Security, LLC for the National Nuclear Security Administration of U.S. Department of Energy under contract 89233218CNA000001. By approving this article, the publisher recognizes that the U.S. Government retains nonexclusive, royalty-free license to publish or reproduce the published form of this contribution, or to allow others to do so, for U.S. Government purposes. Los Alamos National Laboratory requests that the publisher identify this article as work performed under the auspices of the U.S. Department of Energy. Los Alamos National Laboratory strongly supports academic freedom and a researcher's right to publish; as an institution, however, the Laboratory does not endorse the viewpoint of a publication or guarantee its technical correctness. 


\section{INECP Country Engagement Plans and}

\section{Transformational Events}

\section{(8) Los Alamos NATIONAL LABORATORY}

Los Alamos National Laboratory Shane Gonzalez Keepin School Lightning Presentation Student Symposium 


\section{Shane Gonzalez (Groups NEN-1 and NEN-3)}

- Diplomacy and International Studies

o BS Seton Hall University, 2022

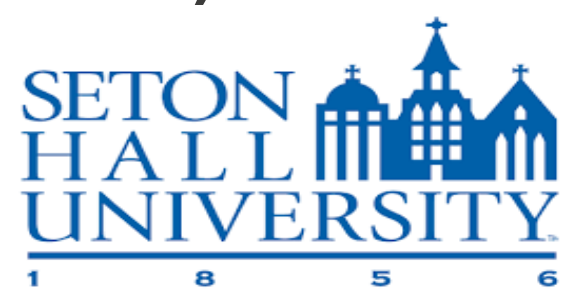

- Division: Nuclear Engineering \& Nonproliferation

- International Threat Reduction (NEN-3) and Safeguards Science and Technology (NEN-1)

○ Mentors:

- NEN-3: Christopher Beedle and Loretta Weiss

- NEN-1: Chloe McMath
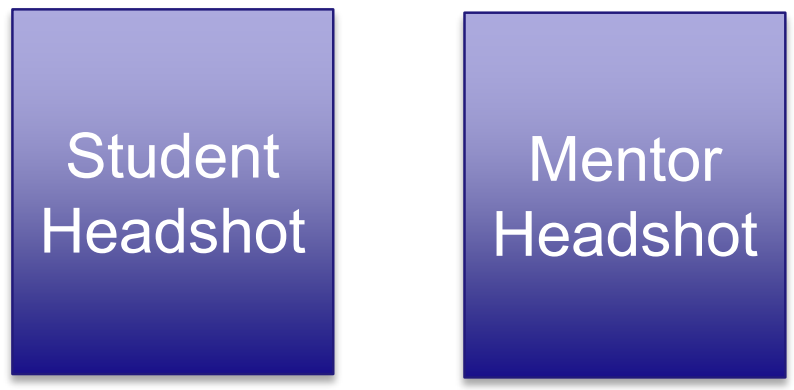

- Research

- Summer Research Topic:

- NEN-3: Updating INECP engagement plans

- NEN-1: Compiling information on transformation events 


\section{Research Overview and Motivation NEN-3}

- What and Why

- The International Nonproliferation Export Control Program (INECP) harnesses the expertise of the U.S. Department of Energy (DOE) national laboratories to assist partners internationally in implementing and adopting strategic trade control measures that can effectively counter WMD procurement efforts.

- INECP's mission is to strengthen global efforts to prevent the illicit or inadvertent transfer of nuclear and dual-use material, equipment, and technology that could contribute to the proliferation of WMD and their means of delivery.

- What am I doing

- Worked with LANL INECP technical leads to update the engagement plan for Turkey, which involved researching Turkey's WMD related legal framework, relevant export control agencies, and WMD-relevant industries (e.g. advanced manufacturing and aerospace). 


\section{Research Overview and Motivation NEN-1 (ongoing)}

- What and Why

- The Emerging Threats and Technologies Working Group under the Office of International Nuclear Security has previously sponsored studies on transformational events that could be exploited to negatively impact nuclear security.

o The purpose of these studies is to identify and develop mitigation strategies for such events.

\section{- What am I doing}

- I am developing a prioritization strategy focusing on specific geographic regions and the transformational events that might affect nuclear security in those areas disproportionately. 


\section{Research Approach}

\section{- How}

- International Nonproliferation Export Control Program (NEN-3)

- Employed open source research techniques

- Document engagement plan sources/references, and compile a reference master list

- Used translation tools

o Safeguards Science and Technology (NEN-1)

- Open source gathering of data on transformation events such as earthquakes or tsunamis. That information is gathered for each of 73 countries. 


\section{Summary of Results}

\section{- Results NEN-3}

- Updated INECP engagement plans

- Learned about Turkey's pharmaceutical, biotechnology, and chemical sectors. (e.g. They have 7684 cultures and world leader in the production of boron chemicals.)

- Got to look at and interpret open source international trade data.

- Learned about Turkey's initiatives regarding research and development of hypersonic technologies such as cruise missiles.

- Learned about Turkey's Commercial Nuclear Sector: e.g. agreements with Russia, China, and Japan to build nuclear power plants.

\section{- Results NEN-1}

o The result of this is a understanding of environmental impacts on areas containing nuclear fuel cycle activities

o It will also offers a understanding of terrorism threats

- Heat maps such as this are used to show area threats to power plants and other infrastructure

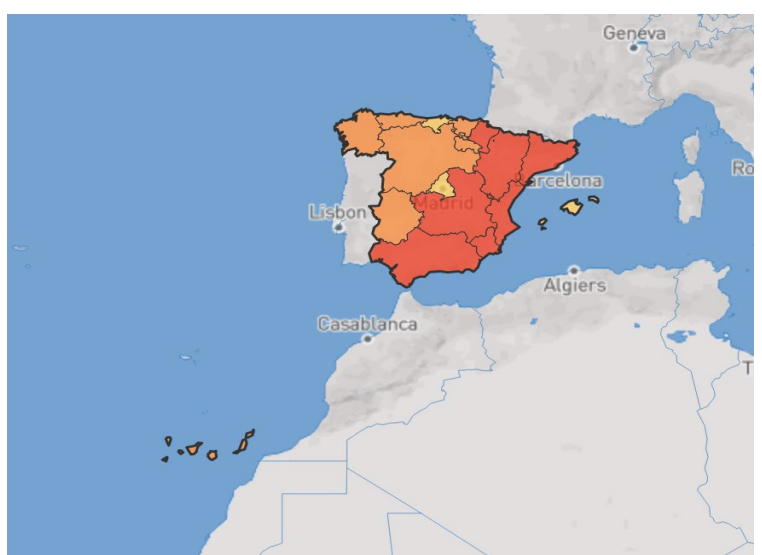

